\title{
Photoluminescence properties of Eu3+-doped low cost zinc silicate based glass ceramics
}

\begin{abstract}

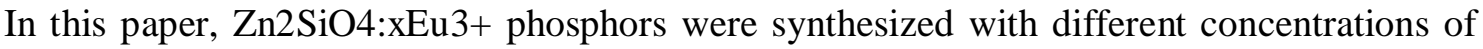
Eu3+ ions $(\mathrm{x}=1,3$ and $5 \mathrm{wt} . \%)$ by using waste bottle glasses as silicate source. The structure, morphology, and luminescent properties of the phosphors were characterized using X-ray diffraction (XRD), field emission scanning electron microscopy (FESEM), and photoluminescence (PL) spectroscopy. The XRD analysis revealed that addition of dopant increased the crystallinity of the samples, and then were decreased dramatically when the dopant concentration further to $5 \mathrm{wt} \%$. The FESEM images showed the samples have irregular in shapes while their emission and excitation peak of Zn2SiO4:xEu3+ phosphor was observed at 600 and $400 \mathrm{~nm}$, respectively.
\end{abstract}

Keyword: Eu3+-doped Zn2SiO4; Solid state method; XRD; Photoluminescence 